\title{
Microcavity top-emitting organic light-emitting devices integrated with microlens arrays: Simultaneous enhancement of quantum efficiency, cd/A efficiency, color performances, and image resolution
}

\author{
Chih-Jen Yang, Su-Hao Liu, Hsing-Hung Hsieh, Chih-Che Liu, \\ Ting-Yi Cho, and Chung-Chih Wu ${ }^{\mathrm{a}}$ \\ Department of Electrical Engineering, Graduate Institute of Photonics and Optoelectronics, \\ and Graduate Institute of Electronics Engineering, National Taiwan University, Taipei, Taiwan 10617, \\ Republic of China
}

(Received 16 October 2007; accepted 1 December 2007; published online 19 December 2007)

\begin{abstract}
A long bothering issue in microcavity organic light-emitting devices (OLEDs) is the difficulty to simultaneously achieve enhanced cd/A efficiency, enhanced external quantum efficiency, enhanced color saturation, and stable colors with viewing angles in the same device design. In this work, we show that microcavity top-emitting OLEDs integrated with microlenses may provide a universal approach for simultaneously achieving all these desired nice characteristics. Furthermore, the pixel blurring often occurring in employment of microlenses to conventional bottom-emitting OLEDs is significantly suppressed by combination of top-emitting microcavity OLEDs and microlenses. (C) 2007 American Institute of Physics. [DOI: 10.1063/1.2827182]
\end{abstract}

Incorporation of the microcavity structure into organic light-emitting devices (OLEDs) is often demonstrated to narrow emission spectra (and thus improve color saturation for display applications) and to enhance the luminance. ${ }^{1-7}$ In some cases, the quantum efficiency of OLEDs can also be enhanced with carefully designed microcavities. ${ }^{2,7}$ However, all these nice characteristics usually do not occur simultaneously for a same microcavity OLED design. Previous theoretical and experimental investigations of microcavity OLEDs had shown that these inconsistent results are mainly associated with settings of microcavity resonant wavelengths. $^{2,7}$ By setting the normal-direction resonant wavelength around the peak wavelength of the intrinsic emission (i.e., photoluminescence, PL) of OLED emitters, one obtains the highest luminance enhancement along the normal direction and negligible color shift with viewing angles, yet accompanied by lower external quantum efficiencies. On the other hand, the highest enhancement in external quantum efficiencies can be obtained by setting the normaldirection resonant wavelength $20-40 \mathrm{~nm}$ longer than the peak wavelength of the intrinsic emission, yet suffering significant color shift over viewing angles. These trade-offs between different emission characteristics (in choosing the resonant wavelength) complicate the design of microcavity devices for different applications. ${ }^{2,7}$

In this paper, we show that by integrating microcavity OLEDs with microlenses, all the enhancements in external quantum efficiency, cd/A efficiency, and color performances (color saturation, small color shift with viewing angles) can be simultaneously achieved. In addition, the image blurring usually accompanying the employment of microlens arrays to OLEDs is also largely reduced by combination of microcavity OLEDs and microlenses. These characteristics may render it universal device architecture for various applications.

\footnotetext{
a) Authors to whom correspondence should be addressed. Electronic mail: chungwu@cc.ee.ntu.edu.tw.
}

The top-emitting microcavity OLEDs without microlenses is shown in Fig. 1(a). The top-emitting microcavity OLED is based on the efficient fluorescent green emitter C545T, ${ }^{3,7,8}$ whose PL shows a peak around $521 \mathrm{~nm}$ and a FWHM (full width at half maximum) of $\sim 60 \mathrm{~nm}$. The device structure is: glass/Ag $(100 \mathrm{~nm}) / m$-MTDATA $: \mathrm{F}_{4}$-TCNQ $\quad(2 \mathrm{wt} \%, 30 \mathrm{~nm}) /$ $\alpha$-NPD $\quad(20 \mathrm{~nm}) / \mathrm{Alq}_{3}: \mathrm{C} 545 \mathrm{~T} \quad(1 \mathrm{wt} \%, 20 \mathrm{~nm}) / \mathrm{Alq}_{3}$ $(40 \mathrm{~nm}) / \mathrm{LiF} \quad(0.5 \mathrm{~nm}) / \mathrm{Al} \quad(1 \mathrm{~nm}) / \mathrm{Ag} \quad(20 \mathrm{~nm}) / \mathrm{ZnSe}$ $(45 \mathrm{~nm}) /$ parylene $(1 \mu \mathrm{m})$. It adopts the high-reflectivity $\mathrm{Ag}$ $(100 \mathrm{~nm})$ as the bottom anode and the thin $\mathrm{Ag}(20 \mathrm{~nm})$ as the semitransparent top cathode. For maximizing optical output of cavity OLEDs, the thin Ag cathode is further capped with a $45 \mathrm{~nm}$ high-index layer of thermally evaporated ZnSe $(n \sim 2.4-2.5)$ to form a low-absorption high-reflection composite mirror for major emission wavelengths of $\mathrm{C} 545 \mathrm{~T}^{3,6,7}$ The organic multilayer structure on top of the $\mathrm{Ag}$ anode in sequence consists of $4,4^{\prime}, 4^{\prime \prime}$-tris(3methylphenylphenylamino) triphenylamine ( $m$-MTDATA) doped with 2 wt $\%$ of tetrafluorotetracyanoquinodimethane $\left(\mathrm{F}_{4}\right.$-TCNQ) as the p-doped hole-injection layer, ${ }^{9}$ $\alpha$-naphthylphenylbiphenyl diamine ( $\alpha$-NPD) as the holetransport layer, ${ }^{3,7,8}$ tris-(8-hydroxyquinoline) aluminum

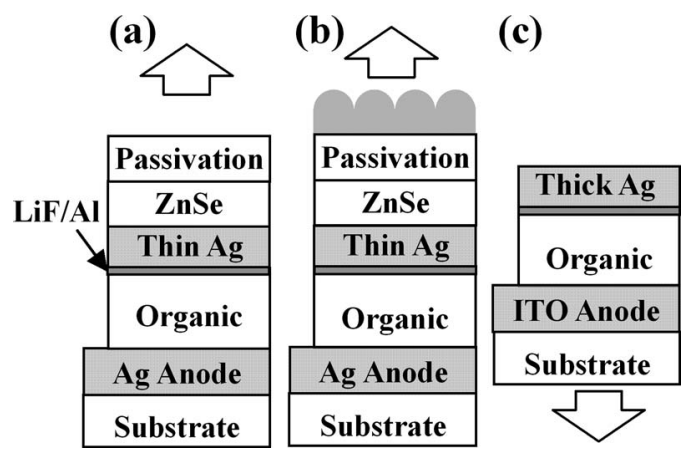

FIG. 1. Device structures of (a) top-emitting microcavity OLED without microlenses; (b) top-emitting microcavity OLED with microlenses; and (c) conventional bottom-emitting OLED. 

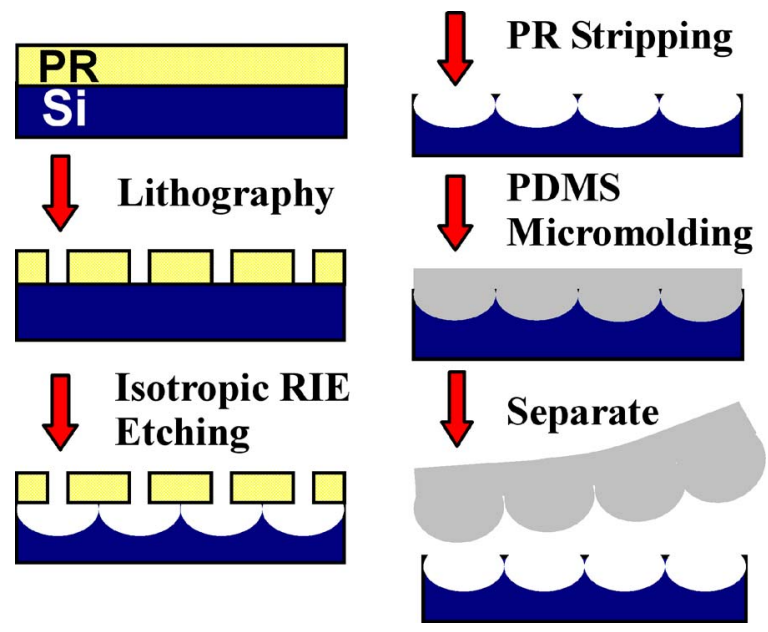

FIG. 2. (Color online) Fabrication processes of the PDMS sheet with microlens arrays.

$\left(\mathrm{Alq}_{3}\right)$ doped with C545T as the emitting layer, ${ }^{3,7,8}$ and undoped $\mathrm{Alq}_{3}$ as the electron-transport layer., ${ }^{3,7}$ Ultrathin layers of $\operatorname{LiF}(0.5 \mathrm{~nm})$ and $\mathrm{Al}(1 \mathrm{~nm})$ serve as the electroninjection layer. ${ }^{3,7,8}$ The micrometer-thick parylene layer is then coated by the room-temperature vapor phase deposition on top of the device as the passivation layer. ${ }^{10}$ The thicknesses of organic layers and the ZnSe layer had been adjusted to set the normal-direction resonant wavelengths around $540 \mathrm{~nm}$ and to locate emitters around the antinode of the cavity. ${ }^{2,3,11}$ The resonant wavelength is set $\sim 20 \mathrm{~nm}$ longer than the PL peak wavelength of C545T to achieve the highest outcoupling and quantum efficiency from the microcavity OLED, according to previous studies. ${ }^{2,7}$ For the topemitting OLED with microlenses [Fig. 1(b)], all the layer structures are the same except that a thin sheet of polydimethylsiloxane (PDMS, $\sim 50 \mu \mathrm{m}$ ) with the microlens array is further laminated on top of parylene. For comparison, a roughly optimized conventional bottom-emitting OLED [Fig. 1(c)] with the structure of glass/indium tin oxide $(120 \mathrm{~nm}) / m$-MTDATA:F - TCNQ $\quad(2 \mathrm{wt} \%, 30 \mathrm{~nm}) /$ $\alpha$-NPD $\quad(20 \mathrm{~nm}) / \mathrm{Alq}_{3}: \mathrm{C} 545 \mathrm{~T} \quad(1 \mathrm{wt} \%, 20 \mathrm{~nm}) / \mathrm{Alq}_{3}$ $(45 \mathrm{~nm}) / \mathrm{LiF}(0.5 \mathrm{~nm}) / \mathrm{Al}(1 \mathrm{~nm}) / \mathrm{Ag}(150 \mathrm{~nm})$ was also tested.

The microlens array on PDMS was fabricated by the micromolding technique using the $\mathrm{Si}$ master mold, ${ }^{12}$ as shown in Fig. 2. The Si master mold was fabricated with the lithography followed by RIE (reactive ion etching). To form the hemispherical concavity, an isotropic RIE recipe $\left(\mathrm{SF}_{6}\right.$, 50 mtorr, RF power $=50 \mathrm{~W}$ ) was used. After stripping the photoresist, PDMS was poured onto the Si mold and was cured at $100{ }^{\circ} \mathrm{C}$ for $30 \mathrm{~min}$. After curing, the PDMS microlens sheet was then lifted for device use. The fabricated thin PDMS sheets $(\sim 50 \mu \mathrm{m}$ thick) with microlens arrays [Fig. $3(\mathrm{a})]$ were examined with the scanning electron microscopy (SEM). The top-view SEM micrograph [Fig. 3(b)] shows the hexagonal arrangement of the microlens array, while the oblique-view SEM micrograph in Fig. 3(c) indicates the nearly hemisphere shape (with diameter of $\sim 10 \mu \mathrm{m}$ ) of the microlenses.

Figures 4(a) and 4(b) show the measured electroluminescence (EL) spectra with relative intensities for the topemitting OLED without and with microlenses, respectively, at viewing angles of $0^{\circ}, 30^{\circ}$, and $60^{\circ}$ off the surface normal, Downloaded 17 Feb 2009 to 140.112 .113 .225 . Redistribution subject

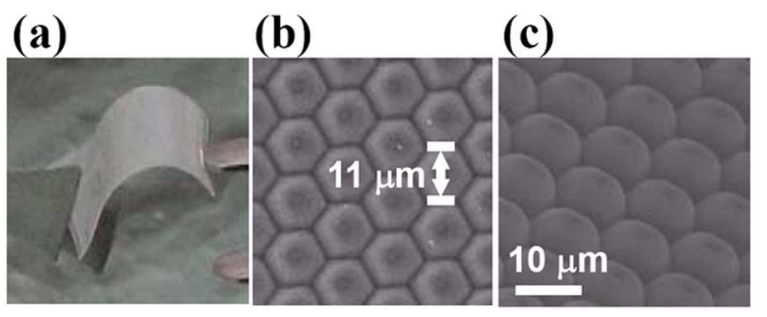

FIG. 3. (Color online) (a) Photo of the PDMS sheet with microlenses arrays; (b) top-view SEM image of the microlenses; (c) oblique-view SEM image of the microlenses.

compared to the (normalized) $0^{\circ} \mathrm{EL}$ spectrum of the conventional bottom-emitting device. Figure 4(c) shows the polar plots of the emission intensities (normalized to the $0^{\circ}$ intensity of the conventional device) for the three devices. Compared to the conventional bottom-emitting device, both top-emitting microcavity devices show narrowed EL spectra and enhanced forward emission intensities. The enhancement of the $0^{\circ}$ intensity is up to $2.5 \times$ and $3 \times$ for top-emitting devices without or with microlenses, respectively. For the top-emitting OLED without microlenses [Fig. 4(a)], the EL shows a sharp emission peak at $538 \mathrm{~nm}$ at $0^{\circ}$ (as expected),
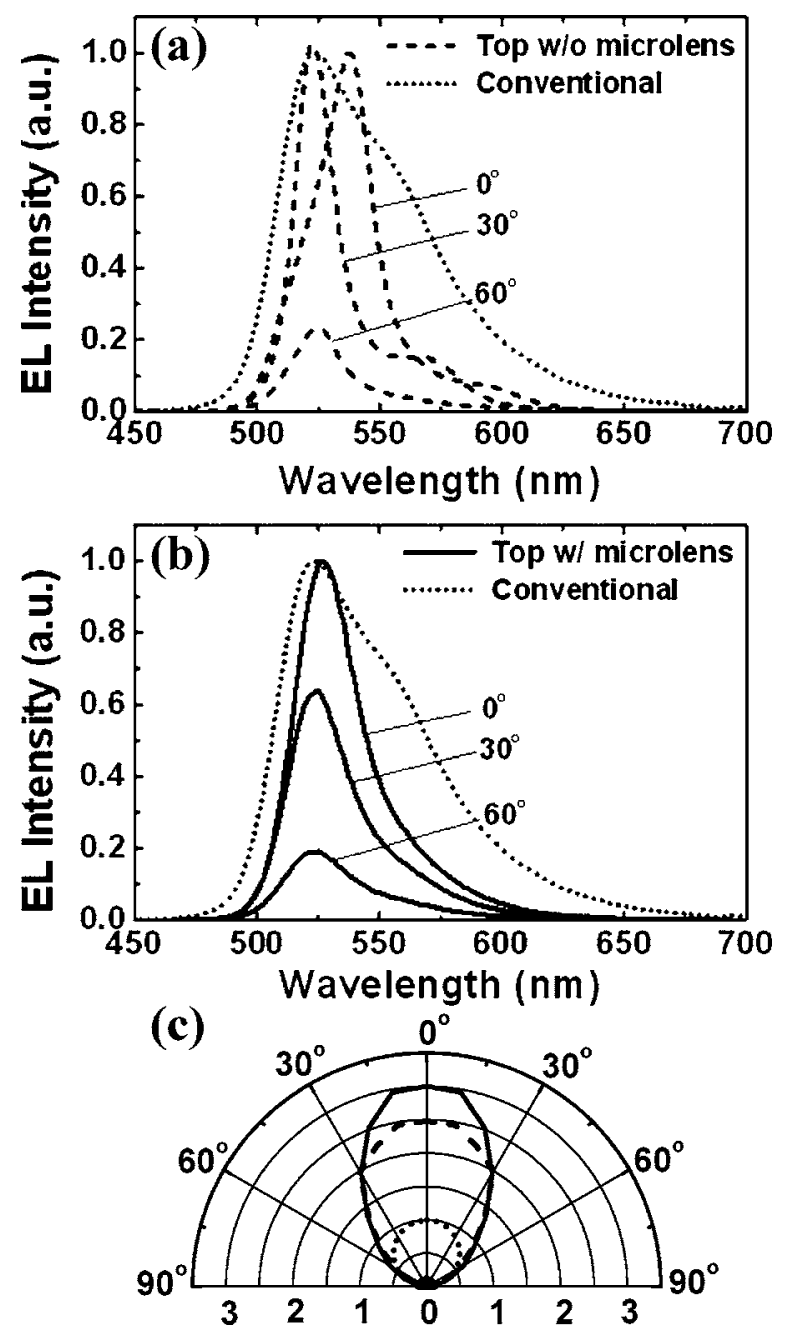

FIG. 4. EL spectra with relative intensities at viewing angles of $0^{\circ}, 30^{\circ}$, and $60^{\circ}$ for top-emitting device (a) without and (b) with microlenses. In (a)-(b), the EL spectrum of the conventional bottom-emitting OLED is also shown for comparison. (c) Polar plots of emission intensities of the three devices (normalized to the $0^{\circ}$ intensity of the conventional bottom-emitting device). 
(a)

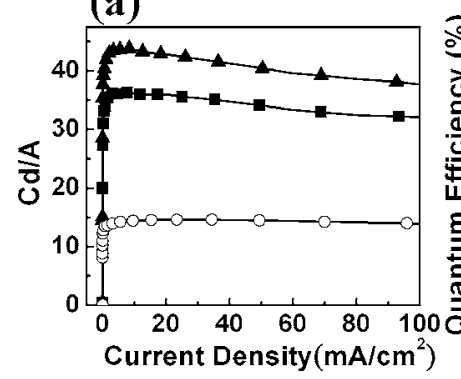

(b)

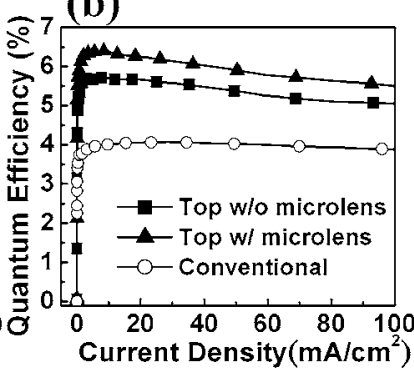

FIG. 5. (a) cd/A efficiencies and (b) quantum efficiencies of the three devices.

and a significant shift to shorter wavelengths with the viewing angles due to strong microcavity effects. Interestingly, with microlens lamination [Fig. 4(b)], the $0^{\circ}$ emission peak slightly blueshifts to $527 \mathrm{~nm}$ and the shift of EL spectra with viewing angles is eliminated. It is also noted that the spectral shapes are modified with microlens lamination. By considering results of Figs. 4(a)-4(c), it may be concluded that lamination of microlenses has led to mixing/averaging EL of different angles and redirecting more light into the forward direction, consequently giving stable/saturated colors over angles and slightly more directed emission (as compared to microcavity OLEDs without microlenses).

Figures 5(a) and 5(b) show the cd/A efficiencies and the external quantum efficiencies, respectively, of the three devices. The conventional bottom-emitting device, the microcavity device without microlenses, and the microcavity device with microlenses show efficiencies up to $(14.5 \mathrm{~cd} / \mathrm{A}$, $4 \%),(36.3 \mathrm{~cd} / \mathrm{A}, 5.7 \%)$, and $(43.8 \mathrm{~cd} / \mathrm{A}, 6.4 \%)$, respectively. As expected, with the resonant wavelength set at $538 \mathrm{~nm}(\sim 20 \mathrm{~nm}$ longer than the PL peak of C545T), the top-emitting microcavity device shows not only enhanced cd/A efficiency $(2.5 \times)$ but also substantially enhanced external quantum efficiency $(1.42 \times),{ }^{2,7}$ as compared to the conventional bottom-emitting device. With microlens lamination, the top-emitting microcavity OLED shows even larger enhancement in both quantum efficiency $(1.6 \times)$ and cd/A efficiency $(3.0 \times)$.

Microlens arrays had been used to enhance the outcoupling efficiencies of conventional bottom-emitting OLEDs. ${ }^{13}$ However, as illustrated in Fig. 6(a), serious image blurring occurs and pixels become hardly distinguishable when the conventional bottom-emitting OLEDs are laminated with microlenses. In contrast, the blurring of pixel emission is substantially reduced for the case of top-emitting OLEDs laminated with microlenses. As illustrated in Fig. 6(b), with microlens lamination, the top-emitting OLED still exhibits a distinguishable pixel edge and clear enough pixel definition for display applications.

Microcavity OLEDs have been useful for enhancing brightness and color saturation of OLEDs. A long bothering

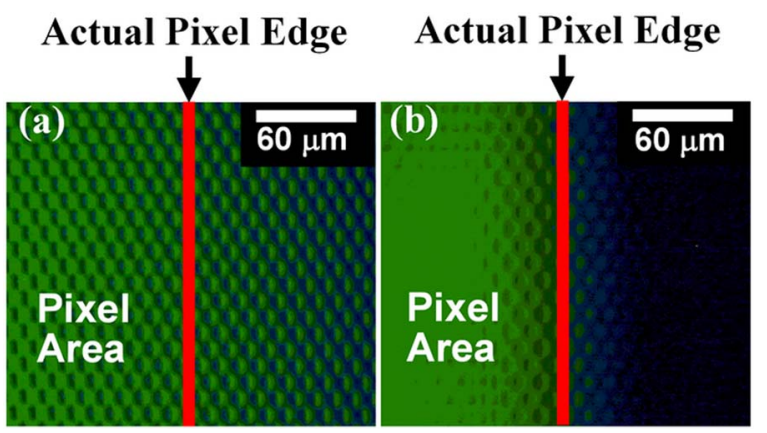

FIG. 6. (Color online) Images of (a) the conventional bottom-emitting device laminated with microlenses and (b) the top-emitting device laminated with microlenses, both taken near the edges of turned-on pixels.

issue in microcavity organic light-emitting devices (OLEDs) is the difficulty to simultaneously achieve enhanced cd/A efficiency, enhanced external quantum efficiency, enhanced color saturation, and stable colors with viewing angles in a same device design. In this work, we show that microcavity top-emitting OLEDs integrated with microlenses may provide a universal approach for simultaneously achieving all these desired nice characteristics. Furthermore, the pixel blurring often occurring in employment of microlenses to conventional bottom-emitting OLEDs is significantly suppressed by combination of top-emitting microcavity OLEDs and microlenses. Since the microlenses can be fabricated separately and then laminated on microcavity OLEDs, the approach reported here is simple, effective, and highly compatible.

The authors would like to acknowledge financial support from National Science Council and National Taiwan University of Republic of China.

${ }^{1}$ N. Takada, T. Tsutsui, and S. Saito, Appl. Phys. Lett. 63, 2032 (1993).

${ }^{2}$ R. H. Jordan, L. J. Rothberg, A. Dodabalapur, and R. E. Slusher, Appl. Phys. Lett. 69, 1997 (1996).

${ }^{3}$ C.-L. Lin, H.-W. Lin, and C.-C. Wu, Appl. Phys. Lett. 87, 021101 (2005).

${ }^{4}$ C.-C. Wu, C.-L. Lin, P.-Y. Hsieh, and H.-H. Chiang, Appl. Phys. Lett. 84, 3966 (2004).

${ }^{5}$ C.-W. Chen, P.-Y. Hsieh, H.-H. Chiang, C.-L. Lin, H.-M. Wu, and C.-C. Wu, Appl. Phys. Lett. 83, 5127 (2003).

${ }^{6}$ H. Riel, S. Karg, T. Beierlein, B. Ruhstaller, and W. Rieß, Appl. Phys. Lett. 82, 466 (2003).

${ }^{7}$ C.-L. Lin, H.-C. Chang, K.-C. Tien, and C.-C. Wu, Appl. Phys. Lett. 90, 071111 (2007).

${ }^{8}$ L. S. Hung, C. W. Tang, M. G. Mason, P. Raychaudhuri, and J. Madathil, Appl. Phys. Lett. 78, 544 (2001).

${ }^{9}$ X. Zhou, M. Pfeiffer, J. Blochwitz, A. Werner, A. Nollau, T. Fritz, and K. Leo, Appl. Phys. Lett. 78, 410 (2001).

${ }^{10}$ K. Yamashita, T. Mori, and T. Mizutani, J. Phys. D 34, 740 (2001).

${ }^{11}$ C.-L. Lin, T.-Y. Cho, C.-H. Chang, and C.-C. Wu, Appl. Phys. Lett. 88, 081114 (2006).

${ }^{12}$ Y. Xia and G. M. Whitesides, Angew. Chem., Int. Ed. 37, 550 (1998).

${ }^{13}$ S. Moller and S. R. Forrest, J. Appl. Phys. 91, 3324 (2002). 